\title{
Why endophenotype development requires families
}

\author{
GLAHN David $\mathrm{C}^{1,2^{*}} \&$ BLANGERO John ${ }^{3}$ \\ ${ }^{1}$ Department of Psychiatry, Yale University School of Medicine, New Haven, CT 06511, USA; \\ ${ }^{2}$ Olin Neuropsychiatry Research Center, Institute of Living, Hartford Hospital, CT 06106, USA; \\ ${ }^{3}$ Department of Genetics, Texas Biomedical Research Institute, San Antonio, TX 78227, USA
}

Received July 8, 2011; accepted July 19, 2011; published online September 2, 2011

\begin{abstract}
Endophenotypes are heritable quantitative traits that are associated with disease liability, can be measured in both affected and unaffected individuals, and provide much greater power to localize and identify risk genes for mental illness than does affection status alone. Traditionally, endophenotypic markers for psychiatric illnesses include in vivo neuroanatomic and functional magnetic resonance imaging measurements and indices of neurocognitive abilities. However, neurocognitive and neuroimaging measures are by no means the only classes of endophenotypes that could be useful for identifying genes for mental illness. Given the advantages of endophenotype-based strategies for elucidating the genetic underpinnings of psychiatric disorders, it would seem prudent to develop a wide range of putative endophenotypes. In order for a measure to be considered a valid endophenotype, it must meet a number of criteria. Specifically, the trait must (1) have moderate to high heritability, (2) be associated with the illness, (3) be independent of clinical state, and (4) impairment must co-segregate with the illness within a family, with non-affected family members showing impairment relative to the general population. While each of these criteria is critical, the heritability and co-segregation requirements are really what differentiate an endophenotype from a simple biomarker. At this time, one requires an experimental design that includes families to demonstrate both heritability and co-segregation. The assertion that novel endophenotypes can not be fully established without family data does not preclude work in unrelated individuals, rather that unrelated samples will only be able to nominate potential candidate endophenotypes that subsequently need to be confirmed in family-based experiments.
\end{abstract}

endophenotype, family studies, biomarker, schizophrenia, bipolar disorder

Citation: Glahn D C, Blangero J. Why endophenotype development requires families. Chinese Sci Bull, 2011, 56: 3382-3384, doi: 10.1007/s11434-011-4740-4

Identification of the molecular mechanisms that increase risk for mental illnesses such as autism, bipolar disorder, major depression, and schizophrenia is of paramount importance. Mental illnesses pose significant economic burdens and are associated with substantial morbidity and mortality rates. However, with very few exceptions, their underlying pathologies are poorly understood. Identifying the specific genes that contribute to risk of these diseases will provide critical information about these illnesses that may lead to the development of novel diagnostic and therapeutic strategies. Although molecular genetic studies of mental diseases have made progress in recent years, bene-

*Corresponding author (email: david.glahn@yale.edu) fiting from a century of genetic epidemiology, the field lags behind those focused on other complex diseases in the identification of disease-related genes and subsequent biological inferences that result from discovering a causal component of the pathological chain. There are many reasons for this, including a historical overdependence on under-powered genetic study designs, as well as the dependence on noisy clinical phenotypes. However, a major reason for the slower pace of progress in the genetics of mental illness is the relative lack of emphasis on the collection and analysis of quantitative allied phenotypes or endophenotypes. Endophenotypes are quantitative traits that are associated with disease liability, are heritable, can be measured in both affected and unaffected individuals, and provide much greater 
power to localize and identify disease-related genes than does affection status alone [1,2]. Using endophenotypic markers may be advantageous since they are thought to be less complex than their associated phenotype and thus may be more readily linked to a specific genetic locus $[1,3]$, as they typically exhibit higher genetic signal-to-noise ratios than diagnosis [4]. In addition, endophenotypes for complex human psychiatric disorders could be potentially extended to animal models [5], advancing our understanding of the neurobiology of psychiatric disorders, and furthering the development of novel medications [6]. Using endophenotypes to identify risk genes for mental illness is consistent with Strategic Objective 1 of the NIMH Strategic Plan: "Promote Discovery in the Brain and Behavioral Sciences to Fuel Research on the Causes of Mental Disorders" (http://www.nimh.nih.gov/about/strategic-planning-reports/i ndex.shtml).

Traditionally, endophenotypic markers for psychiatric illnesses include in vivo neuroanatomic and functional magnetic resonance imaging (MRI) measurements and indices of neurocognitive abilities [7-9]. MRI-based measures of brain structure and function are highly heritable, as we have demonstrated in large extended pedigrees [10,11]. Neuroanatomic and neurophysiologic anomalies are found in most psychiatric illnesses, reflecting the underlying psychopathology of these disorders $[9,12]$. Alterations in brain structure and function seen in individuals with psychiatric illnesses typically predate overt symptom expression [13-15]. In addition, many abnormalities associated with illness have been observed in individuals at risk for these same disorders [16-18]. Similar evidence for neurocognitive traits has accumulated, specifically that these traits are highly heritable [19], and are associated with neuropsychiatric illnesses and deficits in unaffected relatives [20,21]. Taken together, these findings suggest that modern neuroimaging and neurocognitive indices are extremely valuable endophenotypes for mental illnesses. However, neurocognitive and neuroimaging (and electrophysiological [22-24]) measures are by no means the only classes of endophenotypes that could be useful for identifying genes for mental illness. Indeed, other areas of perception or information processing (e.g. olfaction function [25]) or blood-based assays (e.g. [26]) could prove important measures of genetic liability for mental illness and help to delineate the genes and gene systems that predispose these illnesses.

Given the advantages of endophenotype-based strategies for elucidating the genetic underpinnings of psychiatric disorders, it would seem prudent to develop a wide range of putative endophenotypes. However, in order for a measure to be considered a valid endophenotype, it must meet a number of criteria. Specifically, the trait must (1) have moderate to high heritability, (2) be associated with the illness, (3) be independent of clinical state, and (4) impairment must co-segregate with the illness within a family, with non-affected family members showing impairment relative to the general population $[1,3,27,28]$. While each of these criteria is critical, we note, importantly, that the heritability and co-segregation requirements are really what differentiate an endophenotype from a simple biomarker (any biological measure influenced by an illness) [5]. In general, endophenotypes can be considered that subset of biomarkers influenced by genetic factors [5], with the implicit assumption that the same genetic factors that are influencing a particular endophenotype also confer risk for a particular illness (e.g. pleiotropy). At this time, one requires an experimental design that includes families (either twin pairs or pedigrees) to demonstrate both heritability and pleiotropy. The assertion that novel endophenotypes cannot be fully established without family data does not preclude work in unrelated individuals, rather that unrelated samples will only be able to nominate potential candidate endophenotypes that subsequently need to be confirmed in family-based experiments. However, the use of measures as allied phenotypes or endophenotypes in large scale gene discovery projects before the quantitative genetic properties of these indices is established seems premature and potentially quite wasteful. While endophenotypes offer a window into the pathophysiology of mental illness that could dramatically improve our understanding of the biology of these debilitating illnesses, the naïve popularity of the term endophenotype could undermine these efforts and derail the scientific community.

1 Gottesman I I, Gould T D. The endophenotype concept in psychiatry: Etymology and strategic intentions. Am J Psychiatry, 2003, 160: 636-645

2 Blangero J, Williams J T, Almasy L. Novel family-based approaches to genetic risk in thrombosis. J Thromb Haemost, 2003, 1: 1391-1397

3 Lenox R H, Gould T D, Manji H K. Endophenotypes in bipolar disorder. Am J Med Genet, 2002, 114: 391-406

4 Bearden C, Freimer N. Endophenotypes for psychiatric disorders: Ready for primetime? Trends Genet, 2006, 22: 306-313

5 Gould T, Gottesman I. Psychiatric endophenotypes and the development of valid animal models. Genes Brain Behav, 2006, 5: 113-119

6 Nestler E, Barrot M, DiLeone R J, et al. Neurobiology of depression. Neuron, 2002, 34: 13-25

7 Glahn D, Almasy L, Blangero J, et al. Adjudicating neurocognitive endophenotypes for schizophrenia. Am J Med Genet B Neuropsychiatr Genet, 2007, 144B: 242-249

8 Glahn D, Almsay L, Barguil M, et al. Neurocognitive endophenotypes for bipolar disorder identified in multiplex multigenerational families. Arch Gen Psychiatry, 2010, 67: 168-177

9 Meyer-Lindenberg A. Neural connectivity as an intermediate phenotype: Brain networks under genetic control. Hum Brain Mapp, 2009, 30: 1938-1946

10 Winkler A, Kochunov P, Blangero J, et al. Cortical thickness or grey matter volume? The importance of selecting the phenotype for imaging genetics studies. Neuroimage, 2010, 53: 1135-1146

11 Glahn D, Winkler A, Kochunov P, et al. Genetic control over the resting brain. Proc Natl Acad Sci USA, 2010, 107: 1223-1228

12 Glahn D, Thompson P, Blangero J. Neuroimaging endophenotypes: Strategies for finding genes influencing brain structure and function. Hum Brain Mapp, 2007, 28: 488-501

13 Harris J M, Whalley H, Yates S, et al. Abnormal cortical folding in high-risk individuals: A predictor of the development of schizophrenia? Biol Psychiatry, 2004, 56: 182-189 
14 Moffat S D, Szekely C A, Zonderman A B, et al. Longitudinal change in hippocampal volume as a function of apolipoprotein $\mathrm{E}$ genotype. Neurology, 2000, 55: 134-136

15 Fleisher A, Grundman M, Jack C R, et al. Sex, apolipoprotein E epsilon 4 status, and hippocampal volume in mild cognitive impairment. Arch Neurol, 2005, 62: 953-957

16 Hill S, De Bellis M, Keshavan M, et al. Right amygdala volume in adolescent and young adult offspring from families at high risk for developing alcoholism. Biol Psychiatry, 2001, 49: 894-905

17 McDonald C, Bullmore E, Sham P, et al. Association of genetic risks for schizophrenia and bipolar disorder with specific and generic brain structural endophenotypes. Arch Gen Psychiatry, 2004, 61: 974-984

18 Cannon T D, Thompson P M, Van Erp T, et al. Cortex mapping reveals regionally specific patterns of genetic and disease-specific gray-matter deficits in twins discordant for schizophrenia. Proc Natl Acad Sci USA, 2002, 99: 3228-3233

19 Greenwood P, Parasuraman R. Normal genetic variation, cognition, and aging. Behav Cogn Neurosci Rev, 2003, 2: 278-306

20 Weiser M, Reichenberg A, Kravitz E, et al. Subtle cognitive dysfunction in nonaffected siblings of individuals affected by nonpsychotic disorders. Biol Psychiatry, 2008, 63: 602-608

21 Greenwood T, Braff D, Light G, et al. Initial heritability analyses of endophenotypic measures for schizophrenia: The consortium on the genetics of schizophrenia. Arch Gen Psychiatry, 2007, 64: 12421250

22 Freedman R, Adler L E, Leonard S. Alternative phenotypes for the complex genetics of schizophrenia. Biol Psychiatry, 1999, 45: 551-558

23 Olincy A, Braff D, Adler L, et al. Inhibition of the P50 cerebral evoked response to repeated auditory stimuli: Results from the consortium on genetics of schizophrenia. Schizophr Res, 2010, 119: 175-182

24 Turetsky B I, Greenwood T, Olincy A, et al. Abnormal auditory N100 amplitude: A heritable endophenotype in first-degree relatives of schizophrenia probands. Biol Psychiatry, 2008, 64: 1051-1059

25 Turetsky B I, Kohler C, Gur R, et al. Olfactory physiological impairment in first-degree relatives of schizophrenia patients. Schizophr Res, 2008, 102: 220-229

26 Kurian S M, Le-Niculescu H, Patel S D, et al. Identification of blood biomarkers for psychosis using convergent functional genomics. Mol Psychiatry, 2011, 16: 37-58

27 Gershon E, Goldin L. Clinical methods in psychiatric genetics. I. Robustness of genetic marker investigative strategies. Acta Psychiatr Scand, 1986, 74: 113-118

28 Leboyer M, Bellivier F, Jouvent R, et al. Psychiatric genetics: Search for phenotypes. Trends Neurosci, 1998, 21: 102-105

Open Access This article is distributed under the terms of the Creative Commons Attribution License which permits any use, distribution, and reproduction in any medium, provided the original author(s) and source are credited. 